\title{
Ageing-associated DNA methylation dynamics are a molecular readout of lifespan variation among mammalian species
}

\author{
Robert Lowe ${ }^{1 * \dagger}$, Carl Barton ${ }^{1 \dagger}$, Christopher A. Jenkins ${ }^{2}$, Christina Ernst ${ }^{3}$, Oliver Forman ${ }^{2}$, \\ Denise S. Fernandez-Twinn ${ }^{4}$, Christoph Bock ${ }^{5,6,7,8}$, Stephen J. Rossiter ${ }^{9}$, Chris G. Faulkes ${ }^{9}$, Susan E. Ozanne ${ }^{4}$, \\ Lutz Walter ${ }^{10}$, Duncan T. Odom ${ }^{3}$, Cathryn Mellersh ${ }^{2}$ and Vardhman K. Rakyan ${ }^{1,11^{*}}$
}

\begin{abstract}
Background: Mammalian species exhibit a wide range of lifespans. To date, a robust and dynamic molecular readout of these lifespan differences has not yet been identified. Recent studies have established the existence of ageing-associated differentially methylated positions (aDMPs) in human and mouse. These are CpG sites at which DNA methylation dynamics show significant correlations with age. We hypothesise that aDMPs are pan-mammalian and are a dynamic molecular readout of lifespan variation among different mammalian species.

Results: A large-scale integrated analysis of aDMPs in six different mammals reveals a strong negative relationship between rate of change of methylation levels at aDMPs and lifespan. This relationship also holds when comparing two different dog breeds with known differences in lifespans. In an ageing cohort of aneuploid mice carrying a complete copy of human chromosome 21, aDMPs accumulate far more rapidly than is seen in human tissues, revealing that DNA methylation at aDMP sites is largely shaped by the nuclear trans-environment and represents a robust molecular readout of the ageing cellular milieu.

Conclusions: Overall, we define the first dynamic molecular readout of lifespan differences among mammalian species and propose that aDMPs will be an invaluable molecular tool for future evolutionary and mechanistic studies aimed at understanding the biological factors that determine lifespan in mammals.
\end{abstract}

Keywords: Ageing, Methylation, Epigenetics

\section{Background}

The large variation in lifespan among different mammalian species is a fascinating yet poorly understood phenomenon. For example, mice, on average, live for only two years, whereas other species such as humans and whales can live for $>100$ years. Thus far, a variety of different factors have been proposed to correlate with mammalian species lifespan such as body mass, metabolic rate and age of menarche (reviewed in [1]).

\footnotetext{
* Correspondence: r.lowe@qmul.ac.uk; v.rakyan@qmul.ac.uk

${ }^{\dagger}$ Equal contributors

${ }^{1}$ The Blizard Institute, Barts and The London School of Medicine and

Dentistry, Queen Mary University of London, London E1 2AT, UK

Full list of author information is available at the end of the article
}

However, in each case there are exceptions leading to confusion. For example, although body mass is positively correlated with lifespan across mammalian species, this relationship is not true within species such as dogs [2]. Furthermore, these factors are not dynamic molecular correlates of lifespan.

Recently, several studies have reported genome-scale profiles of ageing-associated differentially methylated positions (aDMPs) in the human genome - CpG sites at which DNA methylation dynamics shows a significant correlation with age (a few of the papers in this area are listed in the references [3-8]). Currently, aDMPs represent the most accurate known molecular markers of age in humans. aDMPs are not limited to 
humans, with other recent studies showing similar effects in mice [9-14] and whales [15]. In the former, it was also observed that lifespan-altering interventions can change the rate of ageing-associated DNA methylation dynamics at aDMPs [10-14]. Inspired by these recent results, we hypothesised that not only are aDMPs pan-mammalian, but they could also represent the first known dynamic molecular readout of lifespan variation among different mammalian species.

\section{Results}

The rate of change of ageing-associated DNA methylation is faster in the mouse relative to human

We first re-analysed published ageing-associated genomescale methylation datasets for human and mouse. This involved calling ageing-associated differentially methylated positions (aDMPs) from array-based Illumina $450 \mathrm{~K}$ data for 656 human samples from Hannum et al. [3] and Reduced Representation Bisulphite Sequencing (RRBS) data for 153 mice from Petkovich et al. [11] (see 'Methods'). At q-value $<0.01$, we found $172,365 \mathrm{CpGs}$ in human and 43,909 CpGs in the mouse that were called aDMPs. Analysis of conserved $\mathrm{CpG}$ sites only revealed that approximately $70 \%$ were called as aDMPs in both species, suggesting that short-range cis sequence is unlikely to be the only driving factor in determining whether any given CpG site behaves as an aDMP (Fig. 1b). As mice have a considerably shorter lifespan than humans, the ability to detect aDMPs in mice suggests that the rate of DNA methylation change must be considerably faster. For example, in Fig. 1a, the mouse aDMP shows a methylation change of $\sim 50 \%$ that occurs within just 35 weeks. To confirm this was a general feature of detected aDMPs, we calculated the rate of change of methylation per week for

\section{a}
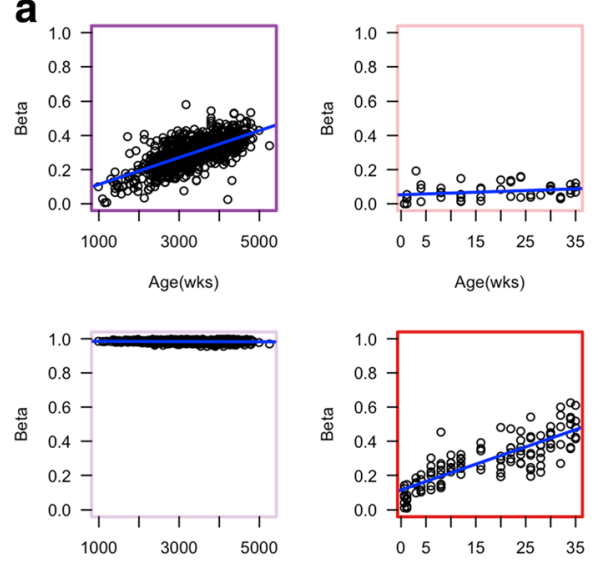

C

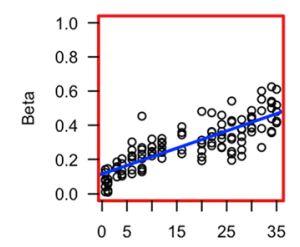

Age(wks)

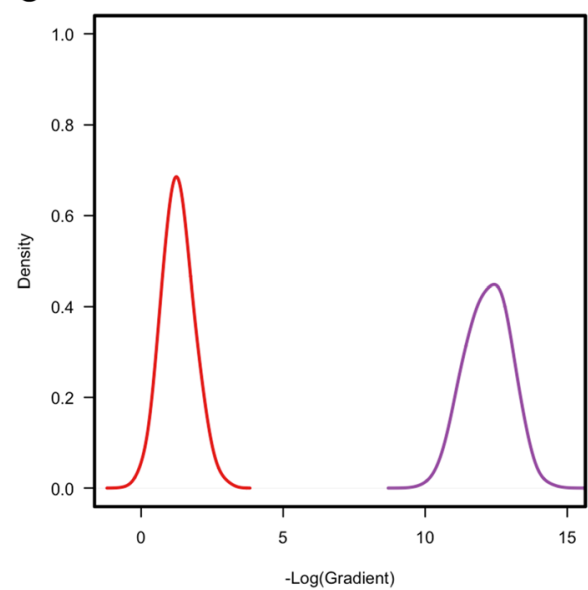

b

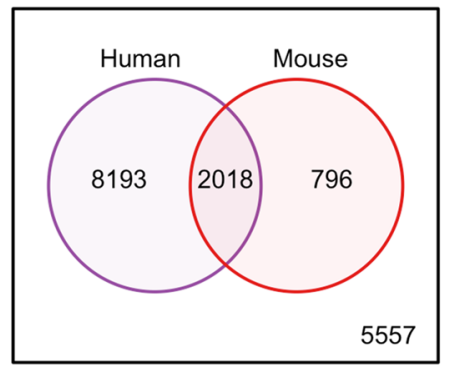

d

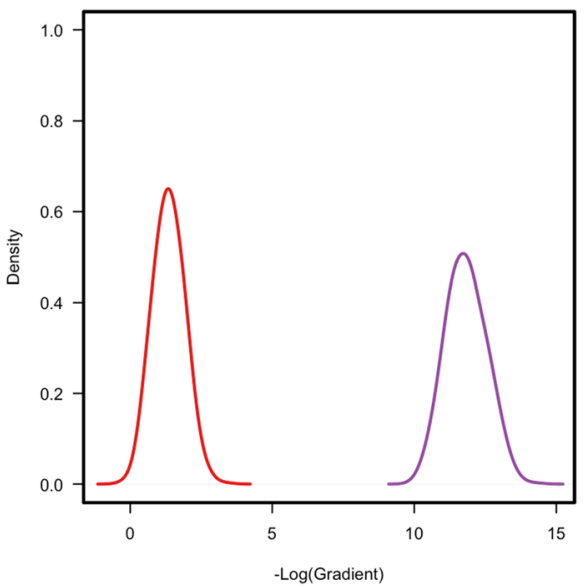

Fig. 1 a Example of called aDMPs. Top: A significant aDMP in human samples (top left) but not in mouse samples (top right). Bottom: A significant aDMP in mouse samples (bottom right) but not in human samples (bottom left). Thick coloured boxes represent a genome-wide significance aDMP in either human (purple) or mouse (red). b A Venn diagram representing the overlap between called aDMPs in mouse and human. c A density plot of the negative log transformed gradients for those aDMPs in non-sequence conserved regions in either mouse (red) or human (purple). $\mathbf{d} A$ density plot of the negative log transformed gradients for those aDMPs in regions showing sequence conservation between mouse (red) and human (purple) 
both human and mouse aDMPs and found that irrespective of whether we use conserved or non-conserved aDMP sites, mouse aDMPs showed a significantly $(P$ value $<2.2$ $\times 10^{-16}$ ) faster rate of change than human aDMPs (Fig. 1c, d), consistent with Stubbs et al. [10]. One potential caveat when comparing species with very different lifespans such as mouse and human is that if there are mouse aDMPs which show similar slow rates of methylation dynamics to those of human aDMPs, they would be difficult to detect as their dynamics would be much too slow to be detected within the lifetime of a mouse. Nevertheless, we can confidently state that at least a significant proportion of aDMPs show considerably different dynamics between a shortlived (mouse) and long-lived (human) mammalian species.

\section{aDMP dynamics are related to mammalian species lifespan}

To extend the above findings, we investigated aDMP methylation dynamics in six different mammalian species spanning a range of documented maximum lifespans $\left(\mathrm{T}_{\max }\right)$, which is a commonly used estimate for the rate of ageing: mouse (Mus musculus, $\mathrm{T}_{\max }=4$ years), dog (Canis familiaris, $\mathrm{T}_{\max }=24$ years), naked mole rat (NMR) (Heterocephalus glaber, $\mathrm{T}_{\max }=31$ ), rhesus macaque (Macaca mulatta, $\mathrm{T}_{\max }=40$ ), humpback whale (Megaptera novaeangliae, $\mathrm{T}_{\max }=95$ ) and human (Homo sapien, $\mathrm{T}_{\max }=$ 122) (all $\mathrm{T}_{\max }$ values are from 'AnAge', http://genomics.senescence.info/species). Due to the high cost of generating sequencing-based deep coverage genome-scale methylation data across many samples, and given that cost-effective commercial DNA methylation arrays are only available for human, we analysed 48 different targeted bisulphite polymerase chain reaction (Bis-PCR) sequencing amplicons for dog and NMR that were chosen based on sequence conservation with human aDMP sites (we show above that human vs mouse differences in methylation ageing rates hold for both conserved and non-conserved aDMP sites). For macaque, due to its close evolutionary distance to human, we generated aDMP profiles using the Illumina $450 \mathrm{~K}$ array (previous studies have successfully used this cost-effective microarray platform for assaying genome-scale methylation differences in a variety of primate species $[16,17])$. For humpback whale, we used existing targeted Bis-PCR sequencing data from Polanowski et al. [15]. For human and mouse, we used previously published genome-scale datasets $[3,14]$. Detailed sample statistics are available in Table 1. For dog, we identified 68 aDMPs that clustered in 15 different targeted aDMP regions (adjusted $P$ value $<0.05$ ) and for NMR we identified $30 \mathrm{aDMPs}$ that clustered in 11 different targeted aDMP regions (adjusted $P$ value $<0.05$ ). For macaque, we determined 29 distinct aDMP regions $(P$ value $<$ $\left.5 \times 10^{-5}\right)$. From each of these CpGs, we determined the rate of dynamic change in methylation levels per week for each species. This yielded a significant negative correlation between rate of change of methylation at aDMP sites and reported $\mathrm{T}_{\text {max }}$ across the different species ( $r$ o $=1, P=$ 0.0028 , Spearman correlation) (Fig. 2a note that in this figure we plot the '-log gradient' of methylation ageing rate). There are four key points to note about these findings: (1) this relationship holds even when comparing mammalian species such as dog, NMR and rhesus macaque, i.e. species with more similar $\mathrm{T}_{\max }$ values to each other, compared with the extreme differences between mouse and human; (2) this relationship is with lifespan per se and not confounded with body mass differences as aDMP methylation dynamics are faster in whales relative to humans even though the former is a much bigger species in terms of mass; (3) our use of different tissues across the different species has negligible influence on the relationship with mammalian lifespan we report here, as analysis of previously published aDMP data from various human tissues reveals that they display similar rates of change with age, and these are significantly greater than between-species differences we report here (Additional file 1: Figure S1); (4) although females show slightly slower ageing-associated methylation dynamics relative to males in human [3], this difference is again smaller than the differences we find among mammalian species.

\section{The correlation between rate of change at aDMPs and lifespan is observed between two different dog breeds}

To examine if the negative correlation between rate of change of methylation at aDMP sites and reported lifespan also holds within a species, we analysed two different dog breeds. Dogs have lived alongside humans for thousands of

Table 1 Sample information for those used in the paper

\begin{tabular}{|c|c|c|c|c|c|c|}
\hline Species & $n$ & Tissue & Age range (weeks) & Reference & Platform & $\overline{\mathrm{aDMRs}(n)^{\mathrm{a}}}$ \\
\hline Human & 656 & Blood & $988-5252$ & Hannum et al. & $450 \mathrm{~K}$ & 256 \\
\hline Mouse & 153 & Whole Blood & $0.67-35$ & Petkovich et al. & RRBS & 2814 \\
\hline Dog & 48 & Buccal & $13-726.96$ & Own & Bis-PCR & 15 \\
\hline NMR & 24 & Liver & $39-1144$ & Own & Bis-PCR & 11 \\
\hline Macaque & 6 & Blood & $52-1040$ & Own & $450 \mathrm{~K}$ & 29 \\
\hline Humpback whale & 45 & Skin & $2.6-1576.953$ & Polanowski et al. & Qiagen PyroMark assays & 3 \\
\hline Tc1 & 6 & Liver & $8-52$ & Own & $450 \mathrm{~K}$ & - \\
\hline
\end{tabular}

${ }^{\mathrm{a}}$ The number of aDMRs is the number of aDMRs used in Fig. $2 \mathrm{a}$ 

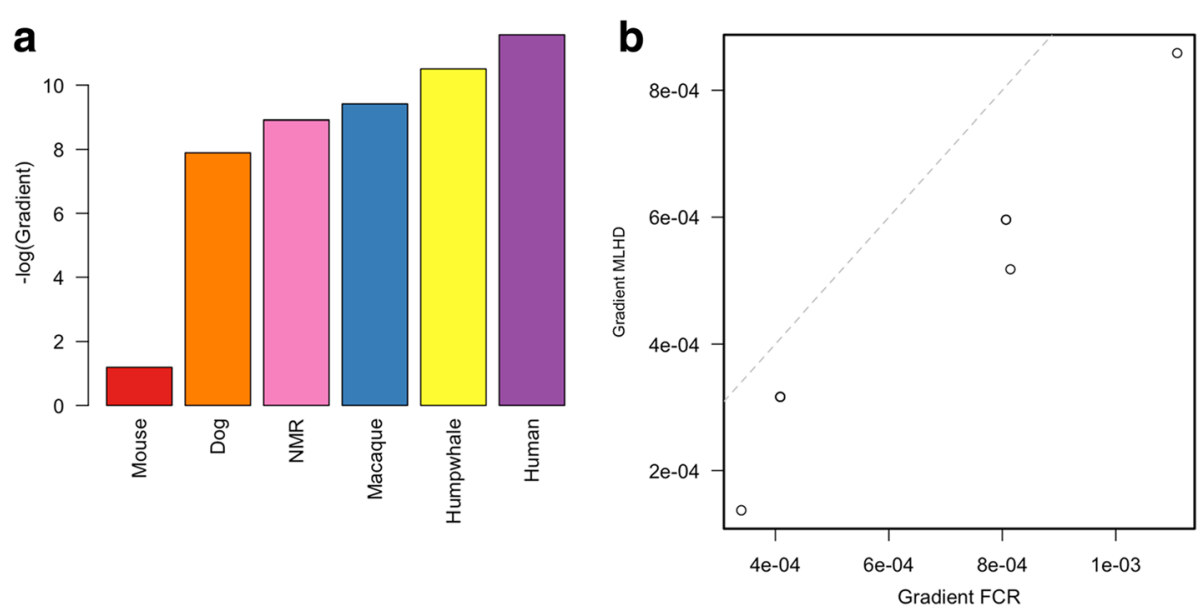

Fig. 2 a A bar plot of the mean negative log gradients for six different species. This shows that the rate of methylation change at aDMPs is proportional to the longevity of the species. $\mathbf{b}$ A plot showing the gradient of significant aDMPs for the two dog breeds profiled (FCR and MLHD). In each of the six aDMPs (two points have similar gradients and overlap each other), the gradient of the FCR is larger than that of the MLHD. Dashed line is the line which represents equal gradient in both breeds, e.g. $y=x$

years and shared similar environmental influences. Artificial selection has led to the generation of $>200$ varieties ('pure breeds') with strikingly different but well characterised phenotypes and attributes, including lifespan which can be studied outside of artificial laboratory conditions. We examined two different pure breeds with contrasting lifespan - the miniature long-haired dachshund (MLHD) (average life expectancy of 12-15 years) and flat-coated retriever (FCR) (average life expectancy of 8-10 years) ([18] and www.thekennelclub.org.uk/pedigreebreedhealthsurvey)).

Only animals that were disease-free at time of sampling were included in our analysis. From the 15 different dog aDMP regions, six regions were identified as aDMPs in both breeds. For all six of these aDMP regions, we found that the shorter-lived FCR showed a significantly faster rate of change of methylation relative to the longer-lived MLHD $(P$ value $=0.0068$, Wilcoxon rank-sum test $)$ (Fig. $2 \mathrm{~b})$. This difference remains unchanged even after removing animals that were aged $<2$ years (a conservative estimate of sexual maturity in dogs). Overall, this provides an example of the negative relationship between rate of change of methylation at aDMP sites and lifespan within a mammalian species.

\section{Rate of change of aDMPs is related to the cellular milieu}

Given the lack of conserved aDMPs across species, it is unlikely that short-range cis-sequence plays a major role. Rather, we hypothesised that the ageing cellular environment per se influences aDMP methylation dynamics. Since this cannot be addressed just by comparing different mammalian species, we used a transchromosomic mouse strain 'Tc1' - that harbours a freely segregating and largely intact functional human chromosome 21 (h-chr21) [19]. We profiled the DNA methylation of 8- to 12-week-old and 44- to 52-week-old Tc1 mice using the human Illumina $450 \mathrm{~K}$ array. This allowed us to determine the methylation state of $3158 \mathrm{CpGs}$ on h-chr21. We restricted our analysis to hchr21 probes that have no significant sequence similarity in the mouse, hence minimising any cross-hybridisation artefacts associated with aDMPs. Furthermore, it has been previously shown that $13,715 \mathrm{CpGs}$ on the $450 \mathrm{~K}$ array also bind mouse DNA [20]. Since the DNA samples only contained human DNA from chromosome 21, we removed those probes from human chromosome 21 that also robustly bind mouse DNA, allowing us to confidently assay the methylation state of 12,358 CpGs in the mouse genome and thus permitting a valuable control analyses. A number of previous studies have shown that the majority of functional attributes of h-chr21in the Tc1 mouse are similar to those found in human cells [21]. Indeed, we confirmed that both mouse and h-chr21CpGs showed lower methylation levels in CpG Islands and promoters compared to open sea, gene bodies and intergenic regions (Fig. $3 \mathrm{a}, \mathrm{b}$ ). Given the relatively small number of Tc1 mice available for our analyses, we did not have the statistical power to perform de novo aDMP calling, but we were able to compare previously reported aDMP profiles with methylation differences between young and old TC1 mice. We therefore initially attempted to investigate the aDMP signature on $\mathrm{h}$-chr21 using existing human liver data $(n=117)$ ( $[22]$ and GSE61258) but found only four aDMPs. Therefore, to provide a more robust set of aDMPs we called pan-tissue aDMPs using samples from multiple human tissues $(n=$ 350) [23]. This yielded 15 aDMPs on human chr 21. Analysis of these in the Tc1 mouse revealed a significant correlation of directionality in ageing-associated dynamics between aDMPs on human chr21 in human cells and the corresponding sites on h-chr21 in the Tc1 mouse $(P=$ 0.011, Fig. 3c). More strikingly, fitting a linear model across 

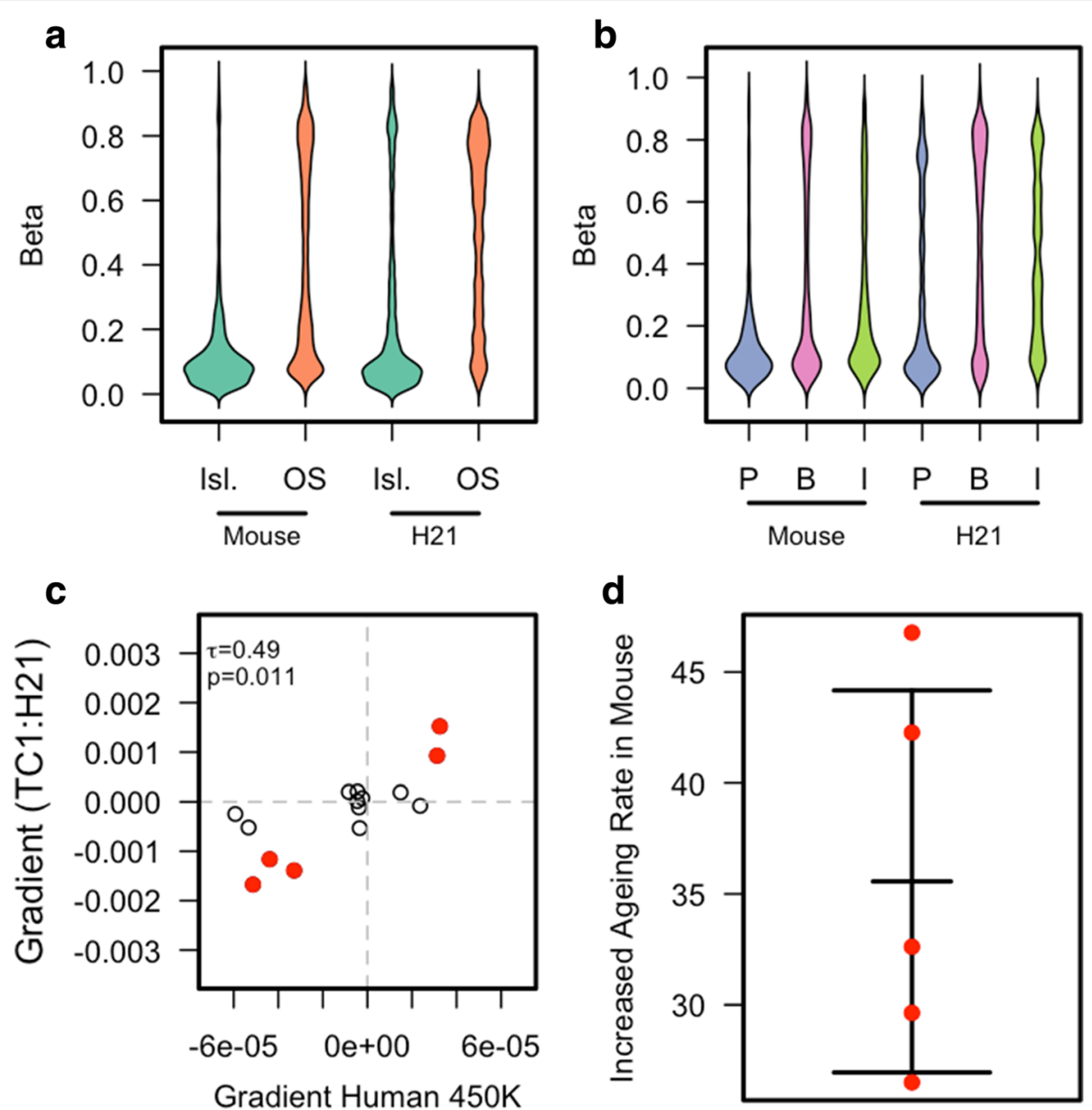

Fig. 3 a A bean plot of the methylation levels for CpG Islands (IsI.) and Open Sea (OS) for both probes matching mouse genome and those uniquely mapping to human chromosome 21. b A bean plot of the methylation levels for promoters (P) (defined as $2 \mathrm{kbp}$ upstream from the TSS), gene bodies (B) and intergenic regions (I) (defined as those probes not either in a promoter or gene body). This is shown for both probes matching mouse genome and those uniquely mapping to human chromosome 21. c A scatter plot of the gradients of human chromosome 21 aDMPs in both human samples and the TC1 mouse. There is a significant correlation of 0.49 ( $P$ value $=0.011)$. Highlighted in red are the probes which show the largest gradient in the TC1 mouse. $\mathbf{d}$ A scatter plot showing the increased rate of methylation change with respect to age in the human chromosome 21 aDMPs in the Tc1 mouse compared to the same aDMP in human samples

all 15 points revealed that the rate of change of methylation with age at these chr21 aDMP sites is approximately 21 times faster in the mouse relative to what is observed in humans (note the differences in scale between the $\mathrm{x}$ - and $\mathrm{y}$ axes in Fig. 3c). Calculating the methylation rate of change for just those aDMPs with the largest change in the Tc1 mouse showed a mean increased rate of $\sim 35$ times that of the same aDMP in humans (Fig. 3d). Therefore, aDMPs on a chromosome from a long-lived species (human) show greatly accelerated methylation dynamics in a short-lived species (mouse), demonstrating that such aDMPs are measuring the rate of cellular ageing.

\section{Discussion and conclusions}

Here we have shown that ageing-associated DNA methylation is a dynamic correlate of lifespan among mammalian species and that these methylation dynamics are measuring cellular ageing as opposed to just chronological age. Crucially, our work shows that, in the context of different mammalian species, the rate of methylation dynamics at aDMPs predict lifespan. That is, aDMPs are more than just a measure of chronological age. Although recent studies have shown that lifespan-altering interventions are associated with changes in dynamics of the epigenetic ageing clock, our work [10-14], in particular the Tc1 experiments, highlights the significant extent to which the dynamics of the clock can be modulated by the transnuclear environment. It is also important to note that in recently published comparisons of human with mouse [10-14] and/or macaque epigenetic clocks [24], it was impossible to determine whether the differences in aDMP dynamics among the different species were due to lifespan or body mass differences. On the other hand, in our manuscript we can categorically state that the rate of the 
epigenetic clock is a measure of lifespan per se and not body mass.

Our work raises two key questions that need to be addressed in future research. First, what is the biological basis of the link between aDMPs and mammalian lifespan? The data from the Tc1 mice show that aDMP dynamics are a consequence of cellular ageing. Ageing comprises a multitude of different processes and one could speculate that the overall aDMP signature reflects information integrated from different sources, as opposed to a single cause. These different upstream processes have stable, cumulative (and measurable) influences on independent subsets of $\mathrm{CpG}$ sites, that collectively represent the aDMP signature. Such a model is also consistent with the idea that of the few known factors thought to influence mammalian lifespan, including body mass, none can solely explain aDMPs. It would be interesting to specifically modulate known signalling pathways to establish how they alter dynamics at specific aDMPs. In this regard, it will be worth exploring whether aDMPs are also found in non-mammalian species that harbour DNA methylation (such as many insects), as these organisms typically have much shorter lifespans and hence provide a more tractable system. Second, it will be important to investigate the functional impact of aDMPs. Within this context, relevant questions include whether there are broader changes in epigenetic state, and/or gene expression, and how such changes might influence ageing-associated processes. Recent large-scale integrative analyses suggest only modest correlations between ageing-associated DNA methylation and gene expression dynamics [3, 23]. This is largely consistent with the complex relationship between DNA methylation and gene expression levels, and it may be that ageing-associated DNA methylation dynamics influence the response of a gene as opposed to steady-state levels. Recent advances in epigenetic engineering methodologies may allow the creation of aDMP signatures in cells (e.g. [25]), thus enabling a more direct assessment of their function including effects on gene expression.

Although future research will undoubtedly address the questions discussed above, it is already clear that aDMPs are a readout of mammalian cellular ageing and, to the best of our knowledge, the first dynamic molecular correlate of lifespan differences among mammalian species. Consequently, they have great potential as molecular markers for studying evolutionary and mechanistic aspects of mammalian ageing.

\section{Methods}

DNA samples

We generated DNA methylation data for dogs, NMRs, macaques and Tc1 mice. All animals did not show any obvious signs of disease at time of sampling. NMRs were maintained at Queen Mary University of London in the Biological
Services Unit, in compliance with institutional guidelines. Macaques were maintained at the German Primate Center, Leibniz-Institute for Primate Research, as a self-sustaining colony of rhesus macaques (Macaca mulatta).

\section{Data processing}

For the 656 samples from Hannum et al., preprocessed samples were downloaded from GEO with accession (GSE40279). For 153 mice from Petkovich et al. [11], methylation and coverage values for each $\mathrm{CpG}$ was obtained from GEO with accession GSE80672. Those CpGs with < $50 \times$ coverage in a minimum of 133 samples were removed from the analysis. For Dog and NMR samples, raw FASTQ files were mapped to the reference canFam 3 and hetGla2, respectively, using BISMARK (v0.16.3) [26] and Bowtie2 (v2.2.8) [27]. Reads that mapped outside of the targeted regions were discarded from analyses and methylated and unmethylated counts for each CpG were calculated using the custom $\mathrm{C}++$ program (https://bitbucket.org/lowelabq$\mathrm{mul} /$ methylation-extractor). Those $\mathrm{CpGs}$ with a coverage < $50 \times$ were also discarded from analyses. For Macaque samples, we first mapped $450 \mathrm{~K}$ probe sequences to the rheMac8 reference genome using BWA [28]. Probes with no mismatch within the first $5 \mathrm{bp}$ and with $<3$ mismatches in total were kept for further analysis (125,102 probes). From these probes, those with a detection $P$ value $>0.01$ were removed and a quantile normalisation on the Red, Green and Type II probes was performed. For TC1 mouse samples, a list of probes previously shown to map to mouse genome was used as well as those probes mapping to h-chr21. Additional filtering was performed to remove probes mapping to h-chr21 which are either deleted or duplicated. Processing was then performed in the same manner as macaque. For Humpback whale, we downloaded methylation values from DRYAD (http://hdl.handle.net/10255/dryad.58061). Data created for this manuscript is available from GEO with accession number GSE86059. Primers used for targeted bisulphite sequencing are listed in Additional file 2.

\section{aDMP calling}

For all aDMP calling, we used the dmpFinder function available in the R package [29] minfi [30] with default parameters, using age in weeks as phenotype variable and continuous as the type. q-values was calculated using the positive false discovery rate [31]. This provided $P$ values and q-values used to filter aDMPs as well as the gradient from the fitted linear model. aDMPs regions were defined by extending each aDMP by $\pm 100 \mathrm{bp}$. Then, overlapping regions are combined into a single region spanning the entire length of the extended aDMPs and the maximum gradient and minimum $P$ value are assigned to the region. Therefore, each region will be a minimum of $200 \mathrm{bp}$ if only a single aDMP is contained in it. 


\section{Sequence conservation analysis}

For comparing between species, we used the UCSC tool liftOver [32] with default parameters except for setting the minimum ratio of bases that must remap to $90 \%$ using the parameter '-minMatch $=0.9$ '. To liftOver $450 \mathrm{k}$ probes, we defined a region of $200 \mathrm{bp}( \pm 100 \mathrm{bp})$ around the target CpG.

\section{Additional files}

Additional file 1: Figure S1. Barplot of the negative log gradient of age in weeks against methylation for multiple different tissues from human samples. (DOCX $113 \mathrm{~kb}$ )

Additional file 2: A table of primer sequences used for targeted assay of methylation. (CSV $3 \mathrm{~kb}$ )

\section{Acknowledgements}

We thank Dr Amelie Kuchler, CeMM Austria for assisting with the preparation of RRBS libraries. This research utilised Queen Mary's Apocrita HPC facility, supported by QMUL Research-IT. https://doi.org/10.5281/zenodo.438045.

\section{Funding}

This research was supported by Cancer Research UK (CE, DTO), the Wellcome Trust (grant 202878/Z/16/Z to DTO) and the European Research Council (grant 615584 to DTO). SEO and DFT are supported by the Medical Research Council (MRC_MC_UU_12012/4). We thank Dr Amelie Kuchler, CeMM Austria for assisting with the preparation of RRBS libraries. This research utilised Queen Mary's Apocrita HPC facility, supported by QMUL Research-IT. https:// doi.org/10.5281/zenodo.438045.

\section{Availability of data and materials}

All data generated in this manuscript are included in NCBI GEO under the accession number GSE86059 [33]. Additional datasets used are previously published and in GEO under accession numbers GSE40279 [34] and GSE80672 [35]. Humpback whale data are found in DRYAD (https://doi.org/ 10.5061/dryad.h4b48) [36]. Human colon and rectal cancer data are from the Genomic Data Commons (https://portal.gdc.cancer.gov) [36].

\section{Authors' contributions}

The study was conceived and coordinated by RL and VKR. DNA samples were provided by CAJ, CE, OF, DSFT, SJR, CGF, SEO, LW, DTO and CM. RRBS libraries were made by CB. Bisulfite PCR was done by VKR. Analysis and interpretation was led by RL, CB and VKR. The manuscript was written by RL and VKR with input from all co-authors. All authors read and approved the final manuscript.

\section{Ethics approval and consent to participate}

The reference number for macaque blood sampling approval is 33.9-425-05$10 A 102$ given by LAVES (Lower Saxony State Office for Consumer Protection and Food Safety). The Tc1 mouse line was housed in the Biological Resources Unit at the Cancer Research UK - Cambridge Institute under Home Office Licence PPL 70/ 7535. This study did not require ethics committee approval for dog analysis as DNA was collected using buccal swabs, which is a non-invasive, non-regulated procedure. No live animals were involved in the research, nor were any in vivo experiments undertaken. All samples were obtained from privately owned pet dogs with the owners' written consent. The majority of DNA samples were obtained using non-invasive buccal/cheek swabs. Where DNA was obtained from blood, these samples were residual aliquots of blood drawn by veterinarians, under the Veterinary Procedures Act, for routine and/or diagnostic veterinary purposes, and not specifically for the purposes of research. NMRs were maintained at Queen Mary University of London in the Biological Services Unit, and tissues obtained from postmortem specimens in compliance with national (Home Office) and institutional procedures and guidelines. Because sample collection was from postmortem material, additional local ethical approval was not required for this study.

\section{Competing interests}

The authors declare that they have no competing interests.

\section{Publisher's Note}

Springer Nature remains neutral with regard to jurisdictional claims in published maps and institutional affiliations.

\section{Author details}

${ }^{1}$ The Blizard Institute, Barts and The London School of Medicine and Dentistry, Queen Mary University of London, London E1 2AT, UK. ${ }^{2}$ Kennel Club Genetics Centre, Animal Health Trust, Newmarket, Suffolk CB8 7UU, UK. ${ }^{3}$ Cancer Research UK Cambridge Institute, University of Cambridge, Cambridge CB2 ORE, UK. ${ }^{4}$ University of Cambridge Metabolic Research Laboratories and MRC Metabolic Diseases Unit, Wellcome Trust-MRC Institute of Metabolic Science, Addenbrooke's Hospital, Cambridge CB2 OQQ, UK. ${ }^{5} \mathrm{CeMM}$ Research Center for Molecular Medicine of the Austrian Academy of Sciences, Vienna, Austria. ${ }^{6}$ Department of Laboratory Medicine, Medical University of Vienna, Vienna, Austria. ${ }^{7}$ Max Planck Institute for Informatics, Saarland Informatics Campus, Saarbrücken, Germany. ${ }^{8}$ Ludwig Boltzmann Institute for Rare and Undiagnosed Diseases, Vienna, Austria. ${ }^{9}$ School of Biological \& Chemical Sciences, Queen Mary University of London, Mile End Road, London E1 4NS, UK. ${ }^{10}$ Primate Genetics Laboratory, Leibniz Institute for Primate Research, German Primate Center, Göttingen, Germany. ${ }^{11}$ Centre for Genomic Health, Queen Mary University of London, EC1M 6BQ, London, UK.

Received: 27 September 2017 Accepted: 19 January 2018

Published online: 16 February 2018

\section{References}

1. Austad SN. J Gerontol A Biol Sci Med Sci. 2009;64(2):199-201.

2. Patronek GJ, Waters DJ, Glickman LT. Comparative longevity of pet dogs and humans: Implications for gerontology research. J Gerontol Biol Sci. 1997;52A:B171-8.

3. Hannum G, Guinney J, Zhao L, Zhang L, Hughes G, Sadda S, et al. Genomewide methylation profiles reveal quantitative views of human aging rates. Mol Cell. 2013;49(2):359-67.

4. Horvath S. DNA methylation age of human tissues and cell types. Genome Biol. 2013;14(10):R115.

5. Rakyan VK, Down TA, Maslau S, Andrew T, Yang TP, Beyan H, et al. Human aging-associated DNA hypermethylation occurs preferentially at bivalent chromatin domains. Genome Res. 2010;20(4):434-9.

6. Teschendorff AE, Menon U, Gentry-Maharaj A, Ramus SJ, Weisenberger DJ, Shen $\mathrm{H}$, et al. Age-dependent DNA methylation of genes that are suppressed in stem cells is a hallmark of cancer. Genome Res. 2010;20(4):440-6.

7. Bell JT, Tsai P-C, Yang T-P, Pidsley R, Nisbet J, Glass D, et al. Epigenome-wide scans identify differentially methylated regions for age and age-related phenotypes in a healthy ageing population. PLoS Genet. 2012;8:e1002629.

8. Heyn H, Li N, Ferreira HJ, Moran S, Pisano DG, Gomez A, et al. Distinct DNA methylomes of newborns and centenarians. Proc Natl Acad Sci U S A. 2012; 109:10522-7.

9. Spiers H, Hannon E, Wells S, Williams B, Fernandes C, Mill J. Age-associated changes in DNA methylation across multiple tissues in an inbred mouse model. Mech Ageing Dev. 2016;154:20-3.

10. Stubbs TM, Bonder MJ, Stark AK, Krueger F, BI Ageing Clock Team, von Meyenn $F$, et al. Multi-tissue DNA methylation age predictor in mouse. Genome Biol. 2017;18(1):68.

11. Petkovich DA, Podolskiy DI, Lobanov AV, Lee SG, Miller RA, Gladyshev VN, et al. Cell Metab. 2017;25(4):954-60. e6

12. Wang T, Tsui B, Kreisberg JF, Robertson NA, Gross AM, Yu MK, et al. Epigenetic aging signatures in mice livers are slowed by dwarfism, calorie restriction and rapamycin treatment. Genome Biol. 2017;18(1):57.

13. Hahn O, Grönke S, Stubbs TM, Ficz G, Hendrich O, Krueger F, et al. Dietary restriction protects from age-associated DNA methylation and induces epigenetic reprogramming of lipid metabolism. Genome Biol. 2017;18(1):56.

14. Cole JJ, Robertson NA, Rather MI, Thomson JP, McBryan T, Sproul D, et al. Diverse interventions that extend mouse lifespan suppress shared age-associated epigenetic changes at critical gene regulatory regions. Genome Biol. 2017;18(1):58.

15. Polanowski AM, Robbins J, Chandler D, Jarman SN. Epigenetic estimation of age in humpback whales. Mol Ecol Resour. 2014;14(5):976-87. 
16. Pai AA, Bell JT, Marioni JC, Pritchard JK, Gilad Y. A genome-wide study of DNA methylation patterns and gene expression levels in multiple human and Chimpanzee tissues. PLoS Genet. 2011;7:e1001316.

17. Hernando-Herraez I, Prado-Martinez J, Garg P, Fernandez-Callejo M, Heyn H, Hvilsom C, et al. Dynamics of DNA methylation in recent human and great apes evolution. PLoS Genet. 2013;9:e1003763.

18. Adams VJ, Evans KM, Sampson J, Wood JL. Methods and mortality results of a health survey of purebred dogs in the UK. J Small Anim Pract. 2010;51(10):512-24.

19. O'Doherty A, Ruf $S$, Mulligan C, Hildreth V, Errington ML, Cooke S, et al. An aneuploid mouse strain carrying human chromosome 21 with Down syndrome phenotypes. Science. 2005;309(5743):2033-7.

20. Wong NC, Ng J, Hall NE, Lunke S, Salmanidis M, Brumatti G, et al. Exploring the utility of human DNA methylation arrays for profiling mouse genomic DNA. Genomics. 2013;102(1):38-46.

21. Wilson MD, Barbosa-Morais NL, Schmidt D, Conboy CM, Vanes L, Tybulewicz $V L$, et al. Species-specific transcription in mice carrying human chromosome 21. Science. 2008;322(5900):434-8.

22. TCGA public repository. http://cancergenome.nih.gov. Accessed 20 July 17.

23. Peters MJ, Joehanes R, Pilling LC, Schurmann C, Conneely KN, Powell J, et al. The transcriptional landscape of age in human peripheral blood. Nat Commun. 2015;6:8570

24. Maegawa S, Lu Y, Tahara T, Lee JT, Madzo J, Liang S, et al. Caloric restriction delays age-related methylation drift. Nat Commun. 2017;8(1):539.

25. Morita S, Noguchi H, Horii T, Nakabayashi K, Kimura M, Okamura K, et al. Targeted DNA demethylation in vivo using dCas9-peptide repeat and scFvTET1 catalytic domain fusions. Nat Biotechnol. 2006;34(10):1060-5.

26. Krueger F, Andrews SR. Bismark: a flexible aligner and methylation caller for Bisulfite-Seq applications. Bioinformatics. 2011;27(11):1571-2.

27. Langmead B, Salzberg S. Fast gapped-read alignment with Bowtie 2. Nat Methods. 2012;9:357-9.

28. Li H, Durbin R. Fast and accurate long-read alignment with BurrowsWheeler transform. Bioinformatics. 2010;26(5):589-95.

29. R Core Team. R: A language and environment for statistical computing, vol. Vienna: R Foundation for Statistical Computing; 2017. https://www.R-project. org/. Accessed 20 July 17.

30. Aryee MJ, Jaffe AE, Corrada-Bravo H, Ladd-Acosta C, Feinberg AP, Hansen $\mathrm{KD}$, et al. Minfi: A flexible and comprehensive Bioconductor package for the analysis of Infinium DNA Methylation microarrays. Bioinformatics. 2014; 30(10):1363-9.

31. Storey JD. The positive false discovery rate: A Bayesian interpretation and the q-value. Ann Stat. 2003;31:2013-35.

32. Hannum G, Guinney J, Zhao L, Zhang L, Hughes G, et al. Genome-wide methylation profiles reveal quantitative views of human aging rates. $\mathrm{Mol}$ Cell. 2013:49:359-67.

33. Lowe R, Barton C, Jenkins CA, Ernst C, Forman O, Fernandez-Twinn DS, et al. Ageing-associated DNA methylation dynamics are a molecular readout of lifespan variation amongst mammalian species. 2018. GEO. GSE86059.

34. Petkovich DA, Podolskiy DI, Lobanov AV, Lee SG, Miller RA, Gladyshev VN. Using DNA methylation profiling to evaluate biological age and longevity interventions. Cell Metab. 2017:25:954-60.

35. Polanowski AM, Robbins J, Chandler D, Jarman SN. Epigenetic estimation of age in humpback whales. Mol Ecol Resour. 2014;14:976-87.

36. Cancer Genome Atlas Network. Comprehensive molecular characterization of human colon and rectal cancer. Genomic Data Commons. https://portal. gdc.cancer.gov. Accessed 20 July 17.

\section{Submit your next manuscript to BioMed Central and we will help you at every step:}

- We accept pre-submission inquiries

- Our selector tool helps you to find the most relevant journal

- We provide round the clock customer support

- Convenient online submission

- Thorough peer review

- Inclusion in PubMed and all major indexing services

- Maximum visibility for your research

Submit your manuscript at www.biomedcentral.com/submit
C) Biomed Central 